\title{
Fabrication of hierarchical moth-eye structures with durable superhydrophobic property for ultra- broadband visual and mid-infrared applications
}

\author{
Litong Dong, ${ }^{1,2,5}$ ZIANG Zhang, ${ }^{3,5}$ LU WANG, $^{1,2}$ ZHANKUN Weng, ${ }^{1,2}$ \\ Mingzhao Ouyang, ${ }^{4}$ Yuegang Fu, ${ }^{4}$ Jiake Wang, ${ }^{4}$ Dayou $L I,{ }^{5}$ and Zuobin
}

WANG ${ }^{1,2,5 *}$

${ }^{1}$ Ministry of Education Key Laboratory for Cross-Scale Micro and Nano Manufacturing, Changchun University of Science and Technology, Changchun 130022, China

${ }^{2}$ International Research Centre for Nano Handling and Manufacturing of China, Changchun University of Science and Technology, Changchun 130022, China.

${ }^{3}$ Changchun Observatory, National Astronomical Observatory, Chinese Academy of Sciences, Changchun 130117, China.

${ }^{4}$ Optics Test Centre, Changchun University of Science and Technology, Changchun 130022, China.

5 IRAC \& JR3CN, University of Bedfordshire, Luton LU1 3JU, UK.

*wangz@cust.edu.cn

Received XX Month XXXX; revised XX Month, XXXX; accepted XX Month XXXX; posted XX Month XXXX (Doc. ID XXXXX); published XX Month XXXX

\begin{abstract}
Multifunctional antireflective coatings have practical applications as important optical components in many fields, especially for optical devices and imaging systems. However, a good antireflection application in the visible region is often unsatisfactory for mid-infrared devices, and the difficulty for obtaining multiple capabilities simultaneously is one of the main factors limiting their applications. In this work, the hierarchical moth-eye structures with superhydrophobicity were fabricated via inductively coupled plasma-reactive ion etching (ICP-RIE) using nanodisc-array masks, which were formed by three-beam laser interference lithography (LIL), for improving the ultra-broadband optical properties. The uniform antireflection efficiency, which was close to $1 \%$-reflectivity covering over the visual and mid-infrared wavelength range, was exhibited by the moth-eye structures with high-quality pillar arrays. Additionally, irregular nanostructures were tailored onto the top of the pillars to generate the hierarchical moth-eye structures for simultaneously obtaining both the superhydrophobic and anticorrosive properties. The fabricated antireflective structures with the features of self-cleaning and durability have the advantage of being for long-term use in harsh environments.
\end{abstract}

() 2018 Optical Society of America under the terms of the OSA Open Access Publishing Agreement

Keywords: ultra-broadband antireflection; mid-infrared; hierarchical moth-eye structures; superhydrophobicity; laser interference lithography.

\section{Introduction}

Biomimetic artificial coatings with broadband antireflective property have attracted tremendous attention as promising building blocks for optical devices and imaging systems, such as displays [1, 2], photovoltaics [3, 4], sensors [5, 6], and stealth [7]. It makes researchers attach importance to self-cleaning and corrosion resistance performances that the active devices are often used in harsh environments including winds, dust storms and acid rains. Various designs of micro- /nano-structures, such as gratings [8], pores [9], nanocones [10], nanotips [11], nanowires [12] and paraboloids [13], have been fabricated to develop antireflective coatings based on the light trapping effect [14-
16] and the effective medium theory (EMT) [17-19]. Brückner et al. [20] reported the sub-wavelength structures with patterned cones displayed less than $2 \%$ reflectance in the range of $3 \mu \mathrm{m}-5 \mu \mathrm{m}$. The ellipsoidal-shaped embossments were usable as masks to nanoimprint motheye structures for a broadband antireflective layer in the mid-infrared [21]. By contrast, colloidal lithography using micro- /nano-structure molds is a beneficial choice to generate ordered antireflective coatings because of its high efficiency and low cost [22, 23]. Besides, wet etching [24] and electrochemical method [25] are usually used to prepared silicon pyramids for photovoltaic applications [26, 27], but the approaches are unfriendly to the environment.

Many applications of optical devices with excellent 
antireflective ability need self-cleaning function to prevent them from being polluted in outdoor environments. Some research groups were inspired by natural animals and plants, and they explored brilliant coatings [28-30]. A typical example was that the lotus leaf was replicated on transparent photopolymers for improving the power of the Si-based photovoltaics [31]. Leem and coworkers [32] fabricated highly-transparent coverglasses with a water contact angle (WCA) of $\sim 112^{\circ}$ for photovoltaic systems. Khan et al. [33] designed antireflective nanostructures with a $141^{\circ}$-WCA for glass-based supporting materials. Besides, the cicada wings were replicated onto polystyrene substrates to attain hydrophobicity and antireflectivity [34]. Zou et al. [35] prepared a raspberry-like particulate layer on different materials by sol-gel technique. The biomimetic coating with ultralow refractive index exhibited outstanding superhydrophobicity and thermal stability. However, due to the difficulty for precise control of structural parameters and obtaining multiple capabilities simultaneously, little work has been reported on the fabrication of ultra-broadband antireflective structures with durable self-cleaning and anticorrosive functions for applications over the visual and mid-infrared spectral region.

In this work, we proposed a hierarchical moth-eye structure, employing the top nano-protrusions and the bottom pillars to obtain multiple capabilities, especially ultra-broadband antireflection. The hexagonally nanodiscarray mask was fabricated by laser interference lithography (LIL). The pillar array was transferred from this mask onto the silicon substrate by inductively coupled plasma-reactive ion etching (ICP-RIE). While forming the moth-eye structures, the nano-protrusions were generated onto the top of pillars. Comparing with the typical pillar-array structures, the hierarchical moth-eye structure resulting from the combination of both possesses the durable surperhydrophobicity and attractive ultra-broadband antireflection over the visual to the mid-infrared region. The experimental results demonstrate that this approach has great potential for harsh environment applications in optoelectronic devices for the visual, near- and mid-infrared wavelength region.

\section{Principle and simulation modeling details}

The optical model is shown in Fig. 1(a) which depicts the light waves $(\lambda)$ falling on the periodic structure with its periodicity larger than the incident wavelength. The multiple internal reflections occur due to the light-trapping effect that the incident light rays are trapped in the textured surface. However, when light interacts with the periodic structure, which has comparable dimensions $(\Lambda<\lambda)$, the light rays tend to bend progressively as the gradient refractive index of rough surface. The effective medium of a periodic structure can be regarded as an inhomogeneous film with the gradient index $n_{\text {eff }}[18,36,37]$, as shown in Fig. 1(b).

In order to minimize the reflectance, the period $\Lambda$ should be smaller the incident wavelength $(\lambda)$ [38], and $\Lambda$ can be expressed as

$$
\Lambda<\frac{\lambda}{\max \left(n_{\text {eff }}\right)+\sin \left(\theta_{\max }\right)},
$$

where $\theta_{\max }$ is the maximum incidence angle of light in the air. The function $n_{\text {eff }}$ is calculated by $[18,36,37]$

$$
f \cdot \frac{n_{s i}{ }^{2}-n_{e f f}{ }^{2}}{n_{s i}{ }^{2}+n_{e f f}{ }^{2}}=\frac{n_{e f f}{ }^{2}-1}{2 n_{e f f}{ }^{2}+1},
$$

where $f$ is the filling factor of the structure, and $f \in(0,1] . n_{s i}$ is the refractive index of silicon. With the refraction index defined, the optical mapping of that is Fig. 1(c). The filling factor is important for controlling the refractive index of the structure.

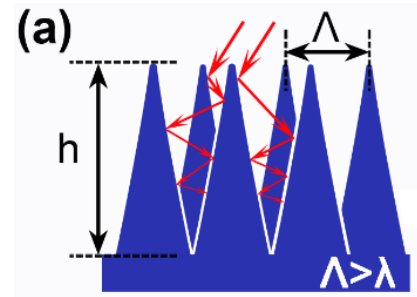

(b)

(c)

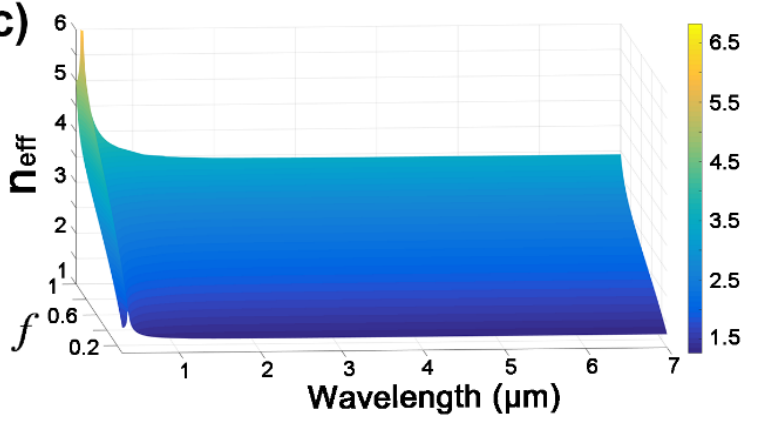

Fig. 1. (a) Light waves undergoing multiple internal reflections through a periodic structure with the height $h$ and period $\Lambda>\lambda$. (b) The geometry of the gradient index layer when $\Lambda<\lambda$. (c) The effective refraction index $n_{\text {eff }}$ of the pillar array as a function of filling factor $(f)$ and wavelength $(\lambda)$.

One of the most important features of antireflective surface-reliefs is the ability to maintain their effectiveness across a wide range of incident wavelengths for many applications. The finite-difference time-domain (FDTD) simulation (Lumerical Solutions Inc.) was employed to simulate the propagation of plane waves in complex structures in the infrared region. Fig. 2(a) represents the moth-eye model with a pillar array, and it also displays the definition of the pattern pitch and pillar height, denoted $\Lambda$ and $h$, respectively. The refraction index of the silicon substrate is about 3.43 in the infrared region [39]. Thus, the refraction index of the periodic structures is in the range of $1.26<n_{\text {eff }}<3.42$, as shown in Fig. 1(c). In general, if the wavelength is $6 \mu \mathrm{m}$ for applications in the infrared region $[21,40,41]$, the period must satisfy Eq. (1): $\Lambda<1.75 \mu \mathrm{m}$. In the experiment, the $1-\mu \mathrm{m}-\Lambda$ pillar-array structure was chosen for the FDTD simulation. The mesh grid of the model was set at $10 \mathrm{~nm}$. For normal incidence, the $\mathrm{x}-$, $\mathrm{y}$ - and z-directions were set as the anti-symmetric boundary, symmetric boundary, and the perfect matched layer (PML) boundary conditions, respectively. In Fig. 2(b), when $h \geq$ $1.50 \mu \mathrm{m}$, the height of the moth-eye structure has little effect on the reflectance in the near- and mid-infrared region. The reflectance is dependent on the $r_{\text {bottom }}$ and $r_{\text {top }}$, respectively, as shown in Figs. 2(c)-(d). Therefore, by mapping the reflectance spectra as the functions of $h, r_{\text {bottom }}, r_{\text {top }}$ and incident wavelength, one promising region in which the modeled structure provides a reflectance lower than $5 \%$ from $1 \mu \mathrm{m}$ to $6 \mu \mathrm{m}$ and it is proved that a pillar $h>2 \mu \mathrm{m}$, $r_{\text {bottom }}>0.40 \mu \mathrm{m}$, and $r_{\text {top }}<0.15 \mu \mathrm{m}$. 

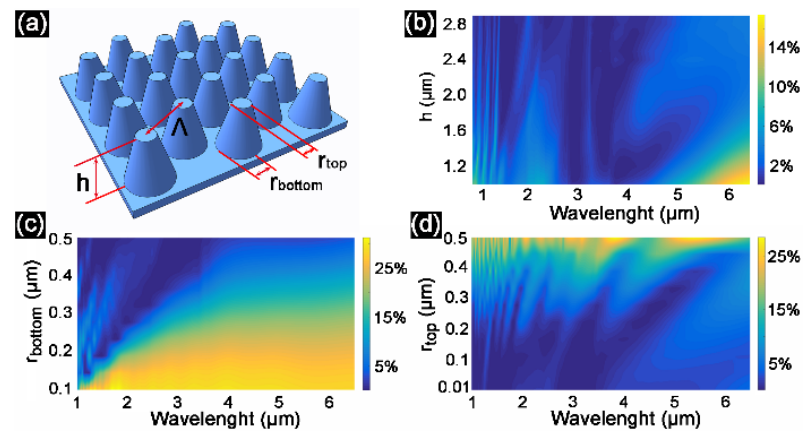

Fig. 2. (a) FDTD model with pillar-shaped moth-eye structures with the protrusion period ( $\Lambda$ ) and height (h). (b) Simulated reflectivity spectra of FDTD model at normal incidence as a function of $h$ when $r_{\text {bottom }}=0.5$ $\mu \mathrm{m}$ and $r_{\text {top }}=0.05 \mu \mathrm{m}$. (c) Simulated reflectivity spectra of FDTD model at normal incidence as a function of $r_{\text {bottom }}$ when $h=2 \mu \mathrm{m}$ and $r_{\text {top }}=0.05$ $\mu \mathrm{m}$. (d) Simulated reflectivity spectra of FDTD model at normal incidence as a function of $r_{\text {top }}$ when $h=2 \mu \mathrm{m}$ and $r_{\text {bottom }}=0.5 \mu \mathrm{m}$.

\section{Experimental methods}

\subsection{Three-beam LIL}

LIL is an effective method for the manufacturing of largearea periodic micro- /nano-structures [42-44]. Multi-beam interference can be described as the superposition of the electric field vectors of coherent beams, and the $m$ th electric field vector is expressed as $\vec{E}_{m}=A_{m} \vec{p}_{m} \cos \left(\vec{k}_{m} \cdot \vec{r}-\omega t+\phi_{m}\right)$, where $A_{m}$ and $\vec{p}_{m}$ are the amplitude and amplitude vector of each plane wave, respectively. $\vec{k}_{m}$ is the wave vector, $\vec{r}$ is the position vector, $\omega$ is the frequency and $\phi_{m}$ is the phase constant. In the three-beam interference field of the TE-TM-TE mode with the corresponding azimuth angles of $0^{\circ}, 90^{\circ}$ and $180^{\circ}$, the three incident angles are $\theta_{1}=\theta_{3}=\theta$ and $\theta_{2}=\theta^{\prime}$, respectively. The simulation result of this three-beam LIL is shown in Fig. 3(a).
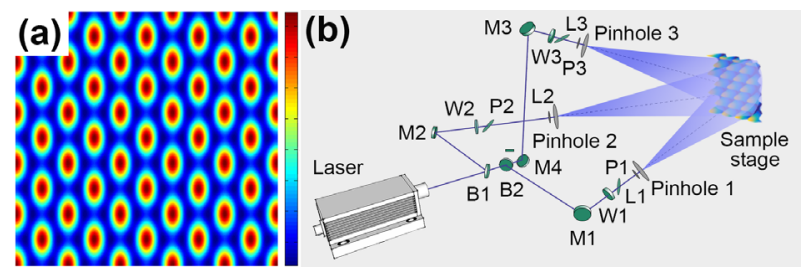

Fig. 3. (a) Simulation pattern of the three-beam interference. (b) Configuration of three-beam LIL system: B - beam splitter, $\mathrm{M}$ - mirror, W - 1/4 wave plate, $\mathrm{P}$ - polarizer, and $\mathrm{L}$ - lens.

In the experiment, the designed interference patterns were obtained by the three-beam LIL system, as shown in Fig. 3(b). The irradiating source was a $360 \mathrm{~nm}$ solid-state laser (MSL-FN-360-S, CNI) with a coherence length over $50 \mathrm{~m}$, and the maximum power of $50 \mathrm{~mW}$. The laser beam was divided into three beams by beam splitters, and the quarter wave plates and the polarizers were placed before a sample to precisely control the powers and the polarization angles of the three beams [44]. The incident angles are $\theta=13.5^{\circ}$ and $\theta^{\prime}=15^{\circ}$, respectively.

\subsection{Materials and Characterizations}

Single-side-polished undoped $\mathrm{Si}<111>$ wafers with the thickness of $500 \mu \mathrm{m}$ were cleaned by acetone, ethanol, and deionized (DI) water, and they were deposited with a 1-nmCr coating that acts as the adhesive layer between the mask and the substrate. The FEI Quanta 250 scanning electron microscope (SEM) was used to observe the surface morphologies of structures. The chemical composition of the samples was analyzed by an energy-dispersive x-ray spectrometer (EDS, Oxford Instruments) detection system. To characterize the antireflective properties of the periodic structures, the specular reflectance of the sample was measured using visible and near-infrared reflectance spectrophotometer (Lambda 950, PerkinElmer) and Fourier-transform infrared spectrometer (Spectrum GX, PerkinElmer) with an incidence angle of $6^{\circ}$. The wettability of the moth-eye structures was carried out on the videobased optical contact angle-measuring system (OCA 25, Dataphysics). The acid $(\mathrm{pH}=1)$ and alkaline $(\mathrm{pH}=13)$ solutions were adjusted by $12 \mathrm{~mol} / \mathrm{L} \mathrm{HCl}$ solution and 10 $\mathrm{mol} / \mathrm{L} \mathrm{NaOH}$ solution at room temperature. The $\mathrm{pH}=7$ droplet was DI water. The $\mathrm{pH}$ values of the solutions were measured by the $\mathrm{pH}$ meter (PB-10, Sartorius).

\subsection{Fabrication of the moth-eye structure}

The moth-eye structure fabrication is outlined in the process flow illustrated in Fig. 4. It begins by spin-coating a silicon wafer with a positive photoresist (AR-P 3740, Allresist) and exposing a hexagonal array pattern (Fig. 4(a)) by the threebeam LIL system, as shown in steps 1 and 2. In step 3, the 60-nm-thick Au coating was deposited on the patterned substrate by sputtering deposition (Q150TES, Quorum Technologies Ltd). After wet chemical stripping the photoresist and lifting off the Au coating, the nanodisc array was left in the designed pattern, as shown in Fig. 4(b). In Fig. 4(c), the SEM image clearly presents that the pillar array has been generated into the silicon substrate followed by ICP-RIE (SI500, Sentech) process (step 4 and 5). During the plasma etching, the residual Au was removed, and the desired moth-eye structure was achieved, like the one shown in Fig. 4(d).

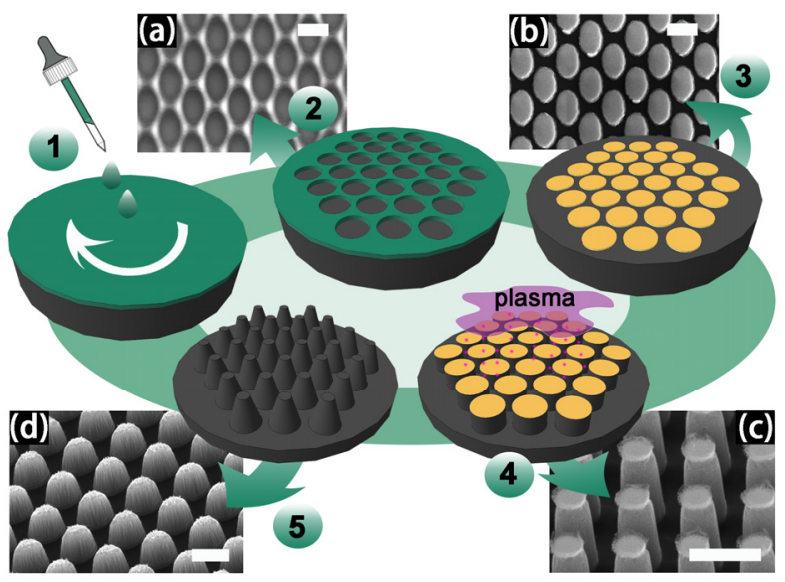

Fig. 4. Schematic diagram of the fabrication process of periodic structures. (1) Spin-coating photoresist; (2) Photoresist pattern by LIL; (3) Depositing Au coating and lift-off; (4-5) Etching Si using Au mask. SEM images at corresponding stages of fabrication: post-lithography (a), post-lift-off (b), and post-plasma etching (c)-(d). Scale bar: $1 \mu \mathrm{m}$.

The silicic surface profile depends on the various experimental conditions such as the composition of gas mixture, RF power and gas pressure. In this experiment, the plasma etching parameters were summarized in Tables 1-2 under the different ways: the durative etching and the cyclic etching. The average values of $h, r_{\text {top }}$ and $r_{\text {bottom }}$ of the 
samples are shown in Table 2.

Table 1. ICP-RIE parameters during the durative etching.

\begin{tabular}{cccccccc}
\hline Sample & $\begin{array}{c}\mathrm{ICP} \text { Power } \\
(\mathrm{W})\end{array}$ & $\begin{array}{c}\mathrm{RF} \text { Power } \\
(\mathrm{W})\end{array}$ & $\begin{array}{c}\mathrm{CHF}_{3} \\
(\mathrm{sccm})\end{array}$ & $\begin{array}{c}\mathrm{SF}_{6} \\
(\mathrm{sccm})\end{array}$ & $\begin{array}{c}\text { Pressure } \\
(\mathrm{mT} \text { Torr })\end{array}$ & $\begin{array}{c}\text { Temperature } \\
\left({ }^{\circ} \mathrm{C}\right)\end{array}$ & $\begin{array}{c}\text { Etching time } \\
(\mathrm{s})\end{array}$ \\
\hline $\mathrm{T} 1$ & 800 & 20 & 60 & 20 & 6 & 20 & 120 \\
$\mathrm{~T} 2$ & 800 & 20 & 60 & 20 & 6 & 20 & 180 \\
$\mathrm{~T} 3$ & 1000 & 20 & 60 & 20 & 7.5 & 20 & 120 \\
\hline
\end{tabular}

Table 2. ICP-RIE parameters during the cyclic etching (one cycle is 30 s) and the structural parameters.

\begin{tabular}{ccccccccccc}
\hline Sample & $\begin{array}{c}\mathrm{ICP} \text { Power } \\
(\mathrm{W})\end{array}$ & $\begin{array}{c}\mathrm{RF} \text { Power } \\
(\mathrm{W})\end{array}$ & $\begin{array}{c}\mathrm{CHF}_{3} \\
(\mathrm{sccm})\end{array}$ & $\begin{array}{c}\mathrm{SF}_{6} \\
(\mathrm{sccm})\end{array}$ & $\begin{array}{c}\text { Pressure } \\
(\mathrm{mT} \text { Torr })\end{array}$ & $\begin{array}{c}\text { Temperature } \\
\left({ }^{\circ} \mathrm{C}\right)\end{array}$ & $\begin{array}{c}\text { Etching } \\
\text { cycle }\end{array}$ & $\begin{array}{c}h \\
(\mu \mathrm{m})\end{array}$ & $\begin{array}{c}r_{\text {top }} \\
(\mu \mathrm{m})\end{array}$ & $\begin{array}{c}r_{\text {bottom }} \\
(\mu \mathrm{m})\end{array}$ \\
\hline $\mathrm{S} 1$ & 800 & 20 & 60 & 20 & 6 & 20 & 5 & 1.50 & 0.30 & 0.45 \\
$\mathrm{~S} 2$ & 800 & 20 & 60 & 20 & 6 & 20 & 6 & 1.80 & 0.20 & 0.43 \\
$\mathrm{~S} 3$ & 800 & 20 & 60 & 20 & 6 & 10 & 6 & 2.00 & 0.15 & 0.43 \\
\hline
\end{tabular}

\section{Results and Discussion}

\subsection{Morphology}

The top-view and tilted-view SEM images of the moth-eye structures are shown in Figs. 5(a)-(i), the corresponding etched parameters are given in Tables 1-2. Generally, the height of pillar increases with the etching time during the ICP-RIE process. Fig. 5(a) shows the pillar with $0.9-\mu \mathrm{m}-$ height was prepared by the plasma etching duration of 120 s. From the residues in Figs. 5(b)-(c), it can be deduced that the height of pillars was about $1.2 \mu \mathrm{m}$ after the etching duration for $180 \mathrm{~s}$. As the increase of continuous etching time for the large-area fabrication of the submicro- and nano-structures, the surface temperature of the sample rose, and the ions accumulated continuously among the sidewalls of the pillars, which causes that the pillars were isotropically etched to taper until the array disappeared. Therefore, reducing the surface temperature of the sample is important to achieve the moth-eye structure with a high aspect ratio (ratio of the height to the width).

In the experiment, the moth-eye structure was successfully transferred from the hexagonal Au-nanodisc array onto the silicon substrate by the cyclic etching of $F$ bases plasma, as shown in Figs. 5(d)-(i). As the cycling times increased during $\mathrm{CHF}_{3} / \mathrm{SF}_{6}$ plasmas, the pillar grew at a rate of $300 \mathrm{~nm} /$ cycle $(10 \mathrm{~nm} / \mathrm{s})$. However, the Au mask was almost completely consumed after 6 cycles of plasma etching, it led to shortening the pillars in the following etching cycles, as shown in Fig. 5(h). Furthermore, the 2$\mu \mathrm{m}$-height pillar array (Figs. 5(f) and (i)) was generated through setting a lower chamber temperature. Notice, it resulted in the silicic nano-protrusion on the top of each pillar due to the Au mask shrank during the last cyclic etching of silicon, as shown in Fig. 5(i).

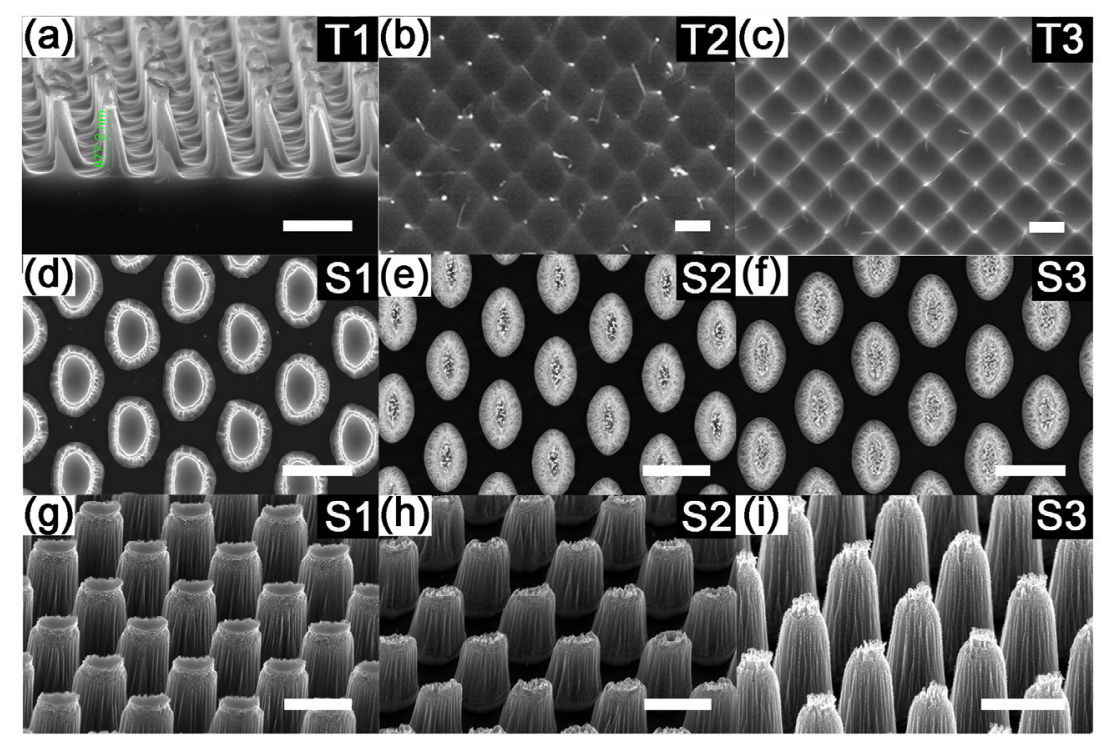

Fig. 5. (a)-(f) Cross-sectional and top-view SEM images of samples. (g)-(i) SEM images of samples (S1, S2 and S3) placed at $45^{\circ}, 30^{\circ}$ and $45^{\circ}$, respectively. Scale bar: $1 \mu \mathrm{m}$.

\subsection{Optical property}

Figs. 6(a)-(b) present the reflectance versus the wavelength for the moth-eye structures (S1, S2 and S3). The antireflective behaviors of the $1-\mu \mathrm{m}-\Lambda$ moth-eye structures occur in the visible wavelength range because of the light- trapping effect [38], as shown in Fig. 1(a). The samples show the same trend in reflectance spectra with the variation of incident wavelength in the visible range. The structures are generated using the same mask, and $r_{\text {bottom }}$ is determined by the mask. However, the different parameters of ICP-RIE etching lead to the changes of $r_{t o p}$ of the moth- 
eye structure, which result in different optical properties in the near-infrared region, as shown in Fig. 6(b). It confirms the observation from the FDTD simulation (Fig. 1(d)) that the reflectance of the structure is strongly dependent on the $r_{t o p}$ of the pillar in the long wavelength region. The spectral curve (the green dotted line) of the sample S1 has three distinct reflection peaks at $1.35 \mu \mathrm{m}, 1.85 \mu \mathrm{m}$ and $2.12 \mu \mathrm{m}$, which is possible due to the smooth top of the pillar array, as shown in Figs. 5(d) and 5(g). The 2- $\mu$ m-height moth-eye structure (S3) shows a good antireflective property in the $0.9-2.5 \mu \mathrm{m}$ wavelength, and its average value is $1.35 \%$.
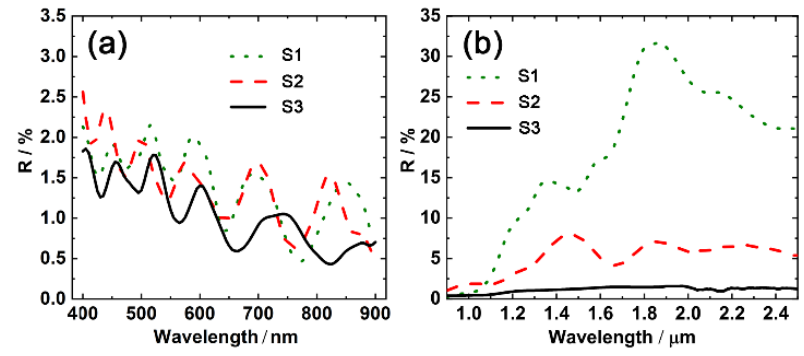

Fig. 6. Optical characterization results of the moth-eye structures in the visible (a) and near-infrared (b) wavelength range.

\subsection{Wettability and anticorrosion characteristics}

The static contact angles of the droplets on the moth-eye structures are shown in Fig. 7(a). It is interesting to see that the pillar array with smooth top morphology is hydrophilic with the WCA of $30^{\circ}$, and the pillar array with nanoprotrusions possesses a WCA with more than $150^{\circ}$. It is known that the wettability is related to the roughness $(\mathrm{R})$ in the Wenzel equation [45]: $\cos \theta_{W}=R \cos \theta_{Y}$, where $\theta_{Y}$ represents the contact angle of a flat surface. Figs. 7(c)-(d) show the irregular nanostructures on the top of the pillars, which demonstrate that the superhydrophobic property of the hierarchical structure is ascribed to the increase of surface roughness. In addition, the moth-eye structure with silicic nano-protrusions also shows stable hydrophobic properties for aqueous $\mathrm{HCl}(\mathrm{pH}=1)$ and aqueous $\mathrm{NaOH}$ $(\mathrm{pH}=13)$.

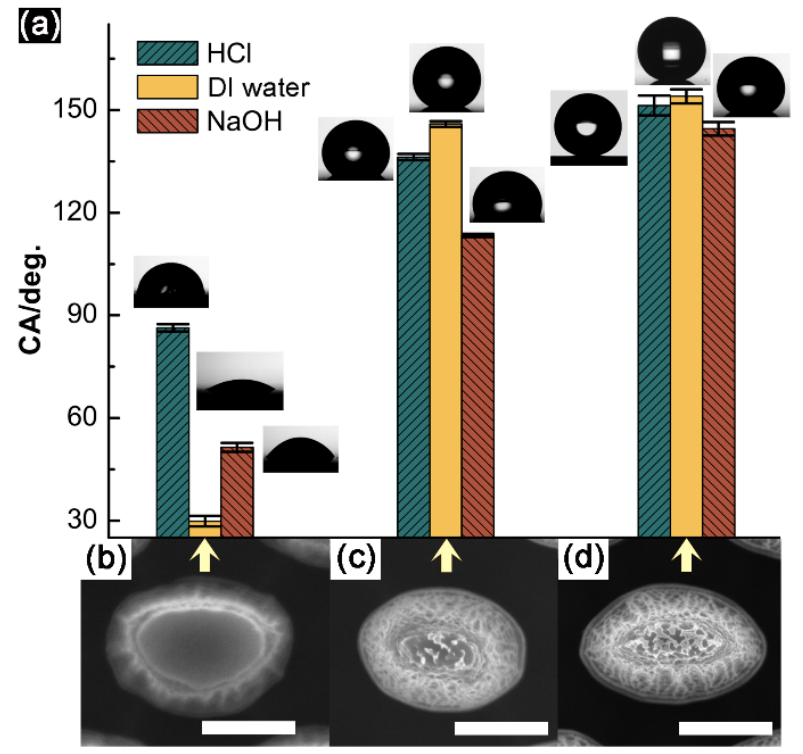

Fig. 7. (a) Static contact angles of the $\mathrm{DI}$ water, $\mathrm{HCl}$ and $\mathrm{NaOH}$ droplets on various substrate surfaces (b-d). The insets correspond to the CA images. Scale bar: $0.5 \mu \mathrm{m}$
The stability and durability of the superhydrophobic surfaces were examined through immersion tests, and the oxygen and silicon were detected. The EDS spectrum of the hierarchical structure is shown in Fig. 8(a). In the tests, the sample (S3) was immersed in $\mathrm{HCl}$, DI water and $\mathrm{NaOH}$ solutions, respectively. Fig. 8(b) shows the variations in the WCAs of the sample's surface with the immersing time. The hierarchical moth-eye structure still exhibited the stable superhydrophobic characteristic after soaking in acid or alkali solutions. The EDS spectra (Figs. 8(c)-(d)) show that the material composition of the hierarchical moth-eye structure is not altered after immersing for 24 hours. $\mathrm{A} \mathrm{SiO}_{2}$ film with the thickness of $2 \sim 5 \mathrm{~nm}$ was coated on the silicon structure exposed in the air, and the film protected the element composition of the moth-eye structure. Clearly, the coating was durable for applications in strong acid and strong alkali environments. Notably, the moth-eye structure exhibits stable superhydrophobic and anticorrosive characteristics, which can maintain the optical properties of the moth-eye structure unaffected from the harsh environments.

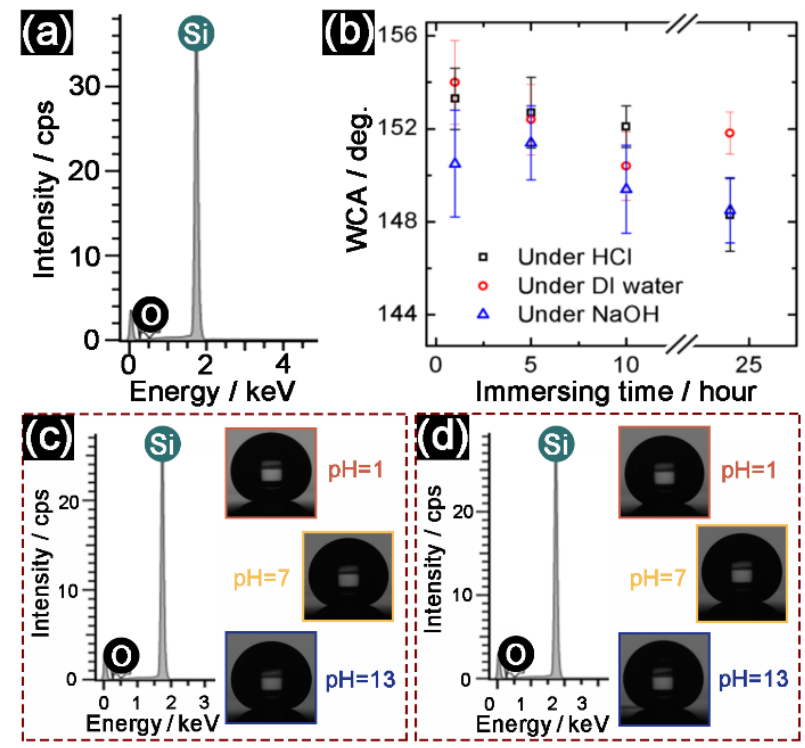

Fig. 8 (a) EDS spectrum of the hierarchical moth-eye structure (sample S3). (b) Variations in the WCAs of the sample S3's surface with the immersing time under $\mathrm{HCl}$, DI water and $\mathrm{NaOH}$ solutions, respectively. (c) and (d) EDS spectra of the hierarchical structure after immersing for 24 hours. The insets show that the contact angles of the $\mathrm{HCl}(\mathrm{pH}=1)$, DI water $(\mathrm{pH}=7)$ and $\mathrm{NaOH}(\mathrm{pH}=13)$ droplets are all about $150^{\circ}$ on the sample' surface.

\subsection{Ultra-broadband antireflection property}

Antireflective coatings with the properties of self-cleaning and durability are important for many applications in harsh environments [46-48]. Fig. 9(a) shows an optical image of droplets on the silicon substrate with an antireflective surface. The droplets of DI water, strong acid and strong alkali exhibit typical spherical shapes on the fabricated surface that is the moth-eye structure with the highly ordered pattern (Fig. 9(b)). From the SEM image of the sample S3 (Fig. 5(i)), there are many nano-ripples on the side walls of pillars, which lead to the dramatic antireflective character in the near-infrared range. Fig. 9(c) shows that the simulated (red curve) and experimental (black curve) reflectance spectra of the hierarchical moth- 
eye structure (sample S3) in air. The light source of spectrophotometer is switched at $2.5 \mu \mathrm{m}$. The measurement result is close to the simulation result in the region from 1 $\mu \mathrm{m}$ to $6 \mu \mathrm{m}$. The designed moth-eye structure shows excellent anti-reflectance performance in the visible and near-infrared regions, and relative reflectance is $\leq 2 \%$ for $\lambda$ $=0.4-6 \mu \mathrm{m}$, while the average reflectance is approximately $1.8 \%$ at the longer wavelength $(\lambda=6-11 \mu \mathrm{m})$.
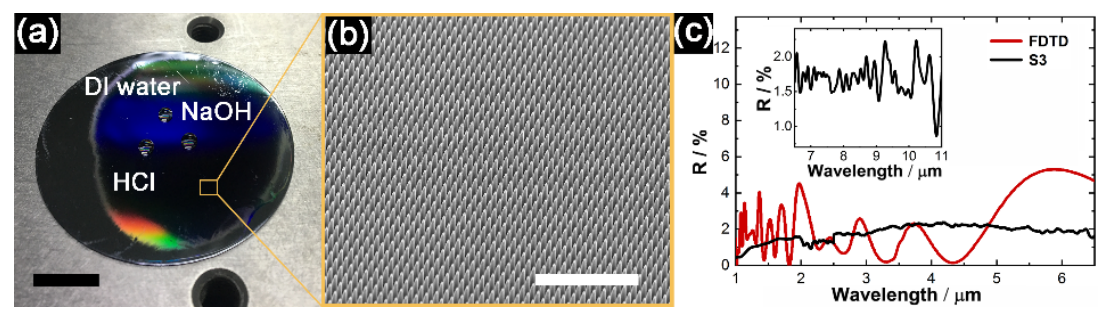

Fig. 9. (a) Photograph of the DI water, $\mathrm{HCl}$ and $\mathrm{NaOH}$ droplets on the sample $\mathrm{S} 3$ (scale bar: $1 \mathrm{~cm}$ ). (b) SEM image corresponding to the sample $\mathrm{S} 3$ (scale bar: $10 \mu \mathrm{m}$ ). (c) The simulated (red curve) and experimental (black curve) reflectance spectra of the hierarchical moth-eye structure. The inset is the reflectance spectrum in the mid-infrared range.

\section{Conclusion}

In summary, we employed a combination of LIL and ICPRIE to fabricate hierarchical moth-eye structures with both the durable superhydrophobicity and the ultra-broadband antireflection. The nanodisc-array masks were generated by three-beam LIL. The highly ordered moth-eye structure was transferred from the nanodisc-array mask onto the silicon substrate by the cyclic etching. As the mask was consumed during plasma etching processes, the nano-protrusions were generated onto the top of pillars to develop the hierarchical moth-eye structure, which improved the self-cleaning and anticorrosive capabilities. Most importantly, the hierarchical moth-eye structure demonstrated the ultrabroadband antireflection properties from the visible to the mid-infrared ranges. The reliable, efficient, and versatile large-area micro/nanofabrication technique developed in

\section{References}

1. G. Tan, J.H. Lee, Y.H. Lan, M.K. Wei, L.H. Peng, I.C. Cheng, and S.T. Wu, "Broadband antireflection film with moth-eye-like structure for flexible display applications," Optica 4(7), 678-683 (2017).

2. W. Yue, S. Gao, S. S. Lee, E. S. Kim, and D. Y. Choi, "Highly reflective subtractive color filters capitalizing on a silicon metasurface integrated with nanostructured aluminum mirrors," Laser Photonics Rev. 11(3), 1600285 (2017).

3. J. K. Kim, S. Chhajed, M. F. Schubert, E. F. Schubert, A. J. Fischer, M. H. Crawford, J. Cho, H. Kim, and C. Sone, "Light - extraction enhancement of GaInN light-emitting diodes by graded - refractive - index indium tin oxide anti - reflection contact," Adv. Mater. 20(4), 801-804 (2008).

4. P. Yu, C. H. Chang, C. H. Chiu, C. S. Yang, J. C. Yu, H. C. Kuo, S. H. Hsu, and Y. C. Chang, "Efficiency enhancement of GaAs photovoltaics employing antireflective indium tin oxide nanocolumns," Adv. Mater. 21(16), 1618-1621 (2009).

5. B. Daglar, T. Khudiyev, G. B. Demirel, F. Buyukserin, and M. Bayindir, "Soft biomimetic tapered nanostructures for large-area antireflective surfaces and SERS sensing," J. Mater. Chem. C 1(47), 7842-7848 (2013).

6. K. M. Rosfjord, J. K. Yang, E. A. Dauler, A. J. Kerman, V. Anant, B. M. Voronov, G. N. Gol’Tsman, and K. K. Berggren, "Nanowire single-photon detector with an integrated optical cavity and anti-reflection coating," Opt. Express 14(2), 527-534 (2006).

7. Z. Diao, M. Kraus, R. Brunner, J.-H. Dirks, and J. P. Spatz, "Nanostructured stealth surfaces for visible and near-infrared light," Nano Lett. 16(10), 6610-6616 (2016).

8. T. Hoshino, M. Itoh, and T. Yatagai, "Antireflective grating in the resonance domain for displays," Appl. Optics 46(5), 648-656 (2007).

9. R.Y. Tsai, C.T. Cheng, C.T. Yang, S.C. Chen, C.C. Huang, S.W. Chen, and W.H. Lu, "Blue laser lithography for making antireflective submicron structures on silicon," Opt. Rev. 20(2), 185-188 (2013).

10. Y. Wang, N. Lu, H. Xu, G. Shi, M. Xu, X. Lin, H. Li, W. Wang, D. Qi, and Y. Lu, "Biomimetic corrugated silicon nanocone arrays for self-cleaning antireflection coatings," Nano Res. 3(7), 520-527 (2010). 
11. Y.F. Huang, S. Chattopadhyay, Y.J. Jen, C.Y. Peng, T.A. Liu, Y.K. Hsu, C.L. Pan, H.C. Lo, C.H. Hsu, and Y.H. Chang, "Improved broadband and quasi-omnidirectional anti-reflection properties with biomimetic silicon nanostructures," Nat. Nanotechnol. 2(12), 770 (2007).

12. E. Garnett, and P. Yang, "Light trapping in silicon nanowire solar cells," Nano Lett. 10(3), 1082-1087 (2010).

13. Y. M. Song, S. J. Jang, J. S. Yu, and Y. T. Lee, "Bioinspired parabola subwavelength structures for improved broadband antireflection," Small 6(9), 984-987 (2010).

14. J. Kr, F. Smole, and M. Topi, "Potential of light trapping in microcrystalline silicon solar cells with textured substrates," Prog. Photovoltaics 11(7), 429-436 (2003).

15. H. Sai, Y. Kanamori, K. Arafune, Y. Ohshita, and M. Yamaguchi, "Light trapping effect of submicron surface textures in crystalline Si solar cells," Prog. Photovoltaics 15(5), 415-423 (2007).

16. C. S. Schuster, S. Morawiec, M. J. Mendes, M. Patrini, E. R. Martins, L. Lewis, I. Crupi, and T. F. Krauss, "Plasmonic and diffractive nanostructures for light trapping-an experimental comparison," Optica 2(3), 194-200 (2015).

17. T. K. Gaylord, W. Baird, and M. Moharam, "Zero-reflectivity high spatial-frequency rectangular-groove dielectric surface-relief gratings," Appl. Optics 25(24), 4562-4567 (1986).

18. D. H. Raguin, and G. M. Morris, "Antireflection structured surfaces for the infrared spectral region," Appl. Optics 32(7), 1154-1167 (1993).

19. P. Lalanne, and D. Lemercier-Lalanne, "On the effective medium theory of subwavelength periodic structures," J. Mod. Optic. 43(10), 2063-2085 (1996).

20. J.B. Brückner, J. Le Rouzo, L. Escoubas, G. Berginc, C. Gourgon, O. Desplats, and J.J. Simon, "Flat-top and patterned-topped cone gratings for visible and mid-infrared antireflective properties," Opt. Express 21(13), 16043-16055 (2013).

21. M. R. Lotz, C. R. Petersen, C. Markos, O. Bang, M. H. Jakobsen, and R. Taboryski, "Direct nanoimprinting of moth-eye structures in chalcogenide glass for broadband antireflection in the mid-infrared," Optica 5(5), 557-563 (2018).

22. Y. Li, J. Zhang, and B. Yang, "Antireflective surfaces based on biomimetic nanopillared arrays," Nano Today 5(2), 117-127 (2010).

23. B. Park, J. Leem, and J. Yu, "Bioinspired Si subwavelength gratings by closely-packed silica nanospheres as etch masks for efficient antireflective surface," Appl. Phys. B 105(2), 335 (2011).

24. Y.J. Hung, S.L. Lee, K.C. Wu, Y. Tai, and Y.T. Pan, "Antireflective silicon surface with vertical-aligned silicon nanowires realized by simple wet chemical etching processes," Opt. Express 19(17), 15792-15802 (2011).

25. X. Ao, X. Tong, D. Sik Kim, L. Zhang, M. Knez, F. Müller, S. He, and V. Schmidt, "Black silicon with controllable macropore array for enhanced photoelectrochemical performance," Appl. Phys. Lett. 101(11), 111901 (2012).

26. T. Rahman, M. Navarro-Cía, and K. Fobelets, "High density micro-pyramids with silicon nanowire array for photovoltaic applications," Nanotechnology 25(48), 485202 (2014).

27. X. Ye, S. Zou, K. Chen, J. Li, J. Huang, F. Cao, X. Wang, L. Zhang, X. F. Wang, and M. Shen, "18.45\% - Efficient multi-crystalline silicon solar cells with novel nanoscale pseudo-pyramid texture," Adv. Funct. Mater. 24(42), 6708-6716 (2014).

28. P. Mazumder, Y. Jiang, D. Baker, A. Carrilero, D. Tulli, D. Infante, A. T. Hunt, and V. Pruneri, "Superomniphobic, transparent, and antireflection surfaces based on hierarchical nanostructures," Nano Lett. 14(8), 4677-4681 (2014).

29. U. Mehmood, F. A. Al-Sulaiman, B. Yilbas, B. Salhi, S. Ahmed, and M. K. Hossain, "Superhydrophobic surfaces with antireflection properties for solar applications: A critical review," Sol. Energ. Mat. Sol. C. 157, 604-623 (2016).

30. M. M. Tavakoli, K.H. Tsui, Q. Zhang, J. He, Y. Yao, D. Li, and Z. Fan, "Highly efficient flexible perovskite solar cells with antireflection and selfcleaning nanostructures," ACS Nano 9(10), 10287-10295 (2015).

31. Z. Huang, C. Cai, L. Kuai, T. Li, M. Huttula, and W. Cao, "Leaf-structure patterning for antireflective and self-cleaning surfaces on Si-based solar cells," Sol. Energy 159, 733-741 (2018).

32. J. W. Leem, X.Y. Guan, M. Choi, and J. S. Yu, "Broadband and omnidirectional highly-transparent coverglasses coated with biomimetic moth-eye nanopatterned polymer films for solar photovoltaic system applications," Sol. Energ. Mat. Sol. C. 134, 45-53 (2015).

33. S. B. Khan, H. Wu, Z. Xie, W. Wang, and Z. Zhang, " $\mathrm{Al}_{2} \mathrm{O}_{3}$ Encapsulated Teflon Nanostructures with High Thermal Stability and Efficient Antireflective Performance," ACS Appl. Mater. Inter. 9(41), 36327-36337 (2017).

34. H. Xie, H.X. Huang, and Y.J. Peng, "Rapid fabrication of bio-inspired nanostructure with hydrophobicity and antireflectivity on polystyrene surface replicating from cicada wings," Nanoscale 9(33), 11951-11958 (2017).

35. X. Zou, C. Tao, K. Yang, F. Yang, H. Lv, L. Yan, H. Yan, Y. Li, Y. Xie, and X. Yuan, "Rational design and fabrication of highly transparent, flexible, 
and thermally stable superhydrophobic coatings from raspberry-like hollow silica nanoparticles," Appl. Surf. Sci. 440, 700-711 (2018).

36. S. Chattopadhyay, Y. Huang, Y.J. Jen, A. Ganguly, K. Chen, and L. Chen, "Anti-reflecting and photonic nanostructures," Mat. Sci. Eng. R. 69(1-3), $1-35(2010)$.

37. H. K. Raut, V. A. Ganesh, A. S. Nair, and S. Ramakrishna, "Anti-reflective coatings: A critical, in-depth review," Energ. Environ. Sci. 4(10), 37793804 (2011).

38. Z. Yu, A. Raman, S. Fan, Fundamental limit of nanophotonic light trapping in solar cells, P. Natl. A. Sci. 107(41), 17491-17496 (2010).

39. D.F. Edwards, E. Ochoa, Infrared refractive index of silicon, Appl. Optics 19(24), 4130-4131 (1980).

40. F. L. Gonzalez, D. E. Morse, and M. J. Gordon, "Importance of diffuse scattering phenomena in moth-eye arrays for broadband infrared applications," Optics Lett. 39(1), 13-16 (2014).

41. D. S. Hobbs, and B. D. MacLeod, "Design, fabrication, and measured performance of anti-reflecting surface textures in infrared transmitting materials," Window and Dome Technologies and Materials IX, 5786, 349-365 (2005).

42. L. Li, M. Hong, M. Schmidt, M. Zhong, A. Malshe, B. Huis, and V. Kovalenko, "Laser nano-manufacturing-state of the art and challenges," CIRP annals 60(2), 735-755 (2011).

43. E. Ertorer, F. Vasefi, J. Keshwah, M. Najiminaini, C. Halfpap, U. Langbein, J. J. Carson, D. W. Hamilton, and S. Mittler, "Large area periodic, systematically changing, multishape nanostructures by laser interference lithography and cell response to these topographies," J. Biomed. Opt. 18(3), 035002 (2013).

44. Z. Zhang, L. Dong, Y. Ding, L. Li, Z. Weng, and Z. Wang, "Micro and nano dual-scale structures fabricated by amplitude modulation in multi-beam laser interference lithography," Opt. Express 25(23), 29135-29142 (2017).

45. $\quad$ R.N. Wenzel, "Resistance of solid surfaces to wetting by water," Ind. Eng. Chem. 8(28), 988-994 (1936).

46. L. Bao, Z. Ji, H. Wang, and R. Chen, "Hollow Rodlike $\mathrm{MgF}_{2}$ with an Ultralow Refractive Index for the Preparation of Multifunctional Antireflective Coatings," Langmuir 33(25), 6240-6247 (2017).

47. J. Son, S. Kundu, L. K. Verma, M. Sakhuja, A. J. Danner, C. S. Bhatia, and H. Yang, "A practical superhydrophilic self cleaning and antireflective surface for outdoor photovoltaic applications," Sol. Energ. Mat. Sol. C. 98, 46-51 (2012).

48. T. Ren, and J. He, "Substrate-versatile approach to robust antireflective and superhydrophobic coatings with excellent self-cleaning property in varied environments," ACS Appl. Mater. Inter. 9(39), 34367-34376 (2017). 